\title{
Sudden death in a patient taking antipsychotic drugs
}

\author{
S H L Thomas, P N Cooper
}

Cardiac arrhythmias are sometimes caused by drugs. One mechanism for drug-induced pro-arrhythmia is delayed ventricular repolarisation, reflected on the surface electrocardiogram (ECG) as prolongation of the QT interval, which is associated with a number of drugs (box 1). Patients with drug-induced QT prolongation are at risk of the polymorphic ventricular tachycardia torsade de pointes. While usually self-limiting, this may degenerate into ventricular fibrillation.

\section{Case summary}

A 68-year-old man with a 5-year history of Alzheimer's disease and cervical spondylosis was admitted to a psychogeriatric ward because of a deterioration in his mental state. $\mathrm{He}$ had no history of heart disease or epilepsy. Because of violent outbursts he was treated with thioridazine $25 \mathrm{mg}$ tid. His other drugs, temazepam 10-30 mg at night, carbamazepine $100 \mathrm{mg}$ bid for neuralgic pain, and droperidol $5-10 \mathrm{mg}$ as required, were unaltered. His behavioural problems initially improved, but 5 days later he was found dead by ward staff, having been observed in his usual state 2 hours earlier.

Post-mortem examination revealed twovessel coronary artery disease including an $80 \%$ stenosis in the left anterior descending artery and a $50 \%$ stenosis in the right coronary artery. However, there was no coronary thrombosis, myocardial infarction, or other significant pathology. The certified cause of death was cardiac arrhythmia due to ischaemic heart

\section{Wolfson Unit of Clinical \\ Pharmacology, University of \\ Newcastle upon Tyne, Newcastle NE2 4HH, UK \\ S H L Thomas \\ Department of Pathology, Royal Victoria Infirmary, Newcastle NE1 4LP, UK \\ P N Cooper}

Accepted 1 October 1997

\section{Examples of drugs which may cause QT prolongation, arrhythmias and sudden death \\ - antidysrrhythmic drugs: Class 1A: quinidine, procainamide, disopyramide; Class III: amiodarone, sotalol \\ - calcium channel blockers: prenylamine ${ }^{\star}$, terodiline ${ }^{\star}$ \\ - psychiatric drugs: thioridazine, pimozide, chlorpromazine \\ - antihistamines: terfenadine, astemizole \\ - antimicrobial and antimalarial drugs: erythromycin, quinine, halofantrine \\ - others: cisapride, tacrolimus \\ ‡ withdrawn after causing serious arrhythmias}

Box 1 disease. Thioridazine was considered as a possible contributing factor and a Yellow Card was sent to the Committee on Safety of Medicines (CSM).

\section{Discussion}

Spontaneous reporting schemes, such as the Yellow Card scheme in the UK, are of value in detecting serious adverse reactions to drugs, but one limitation of these schemes, even for fatal adverse events, is under-reporting. It has been estimated that only $15 \%$ of fatal episodes of thromboembolism in women taking combined oral contraceptives, ${ }^{2}$ and $11 \%$ of fatal blood dyscrasias associated with phenylbutazone or oxyphenbutazone, ${ }^{3}$ were reported by doctors or coroners. One possible reason for this is that a cause and effect relationship may be uncertain. This is particularly true for sudden deaths, because there is no opportunity to observe the effects of drug withdrawal (dechallenge) or rechallenge on the patient. Moreover, patients may have underlying conditions that render them at increased risk of sudden death in the absence of drug therapy, for example, ischaemic heart disease. In these, the risk of sudden death may be increased further by exposure to drugs with arrhythmogenic properties. Unless the death is preceded by features suggestive of drug toxicity such as torsade de pointes, it is impossible to be confident that a sudden death is drug-induced.

This case illustrates many of these difficulties. In favour of this being a drug-induced arrhythmia, the episode occurred soon after the institution of the thioridazine and there was no evidence of acute myocardial infarction or any other cause of death. However, the patient had coronary artery disease which placed him at increased risk of sudden cardiac death in the absence of drug treatment, so coincidence is possible.

Antipsychotic drugs may prolong the QT interval and have been linked to sudden death. Thioridazine, with its prominent quinidine-like actions, has been most commonly implicated. ${ }^{1}$ In Finland, thioridazine was involved in more than half of the sudden deaths affecting patients receiving psychiatric drugs, and this is out of proportion to its use. ${ }^{4}$ The CSM/MCA database for thioridazine includes six reports of unspecified arrhythmia, six of syncope, three of unspecified ventricular arrhythmia, seven of ventricular tachycardia (one torsade de pointes) and 13 of cardiac arrest or ventricular fibrillation. Eleven of these suspected reactions were 


\section{Learning points}

- fatal adverse drug reactions are rare. In spite of their importance, they are under-reported by doctors and coroners

- it may be particularly difficult to be certain of a causal relationship between a drug and a fatal reaction, particularly an arrhythmia.

Nevertheless, all suspected fatal adverse drug reactions should be reported to the CSM

- drugs which prolong ventricular repolarisation, and thus the QT interval on the ECG, may sometimes cause ventricular arrhythmias and sudden death

- the inappropriate use of antipsychotic drugs in the elderly should be avoided, as this group is at increased risk of serious extrapyramidal and cardiovascular adverse effects

Box 2

fatal. However, thioridazine is commonly prescribed and its use is increasing, particularly in frail elderly patients. It is inevitable that a number of sudden deaths will occur coincidentally in recipients. The absolute risk of sudden

1 Thomas SHL. Drugs, QT interval abnormalities and ventricular arrhythmias. Adverse Drug React Toxicol Rev 1994;13:77-102.

2 Inman WHW, Vessey MP, Westerholm B, Engelund A Thromboembolic disease and the steroid content of oral contraceptives. BMJ 1970;2:203-6.

3 Inman WHW. Study of fatal bone marrow depression with special reference to phenylbutazone and oxyphenbutazone. BMJ 1977;1:1500-3.

4 Mehtonen OP, Aranko K, Malkonen L, Vapaatalo H. Survey of sudden death associated with the use of antipsychotic or antidepressant drugs: 49 cases in Finland. Acta Psychiat Scand 1991;84:58-64. death associated with thioridazine therapy is unknown, but likely to be increased in elderly subjects because of their higher prevalence of underlying cardiac disease. Therapeutic doses of carbamazepine therapy have been associated with cardiac conduction defects, ${ }^{5}$ but not arrhythmia or QT interval prolongation. The possibility of an interaction with thioridazine cannot be excluded.

In spite of the problem in determining cause and effect, it is very important to report all fatal and other serious reactions, including those involving old drugs and even if an association is already recognised, as patterns may emerge when several similar reports are received. For example, in 1990 reports of 13 sudden deaths in recipients of the antipsychotic drug pimozide allowed the CSM to identify risk factors and to issue specific recommendations for the safer use of the drug. ${ }^{6}$ Similarly, reports of ventricular arrhythmias, including torsade de pointes, and sudden death with terodiline led to it being withdrawn by the manufacturer in 1991 . $^{7}$

Keywords: adverse drug reaction; thioridazine; cardiac arrhythmias

5 Benassi E, Bo GP, Cocito L, Maffini M, Loeb C. Carbamazepine and cardiac conduction disturbances. Ann Neurol 1987;22:280-1.

6 Committee on Safety of Medicines. Cardiotoxic effects of pimozide. Curr Probl 1990;29.

7 Committee on Safety of Medicines. Withdrawal of terodiline. Curr Probl 1991;32. 